\title{
Developing Space Weather products and services in Europe - Preface to the Special Issue on COST Action ES0803
}

\author{
Anna Belehaki ${ }^{1, *}$, Mauro Messerotti ${ }^{2}$, and Maurizio Candidi ${ }^{3}$ \\ 1 National Observatory of Athens, Institute of Astronomy, Astrophysics, Space Applications and Remote Sensing, \\ Metaxa and Vas. Pavlou, Palaia Penteli 15236, Greece \\ ${ }^{*}$ Corresponding author: belehaki@noa.gr \\ 2 Istituto Nazionale di Astrofisica, Astronomical Observatory of Trieste, Loc. Basovizza n. 302, 34149 Trieste, Italy \\ 3 Istituto Nazionale di Astrofisica, Institute for Astrophysics and Planetology in Space, Via Fosso del Cavaliere 100, 00133 Roma, Italy
}

Received 13 October 2014 / Accepted 27 October 2014

\begin{abstract}
COST Action ES0803 "Developing Space Weather products and services in Europe" primarily aimed at forming an interdisciplinary network among European scientists dealing with different issues relevant to Geospace as well as warning system developers and operators in order to assess existing Space Weather products and recommend new ones. The work that has been implemented from 2008 to 2012 resulted in advances in modeling and predicting Space Weather, in recommendations for the validation of Space Weather models, in proposals for new Space Weather products and services, and in dissemination, training, and outreach activities.

This preface summarizes the most important achievements of this European activity that are detailed in this special issue by the key scientists who participated in COST Action ES0803.
\end{abstract}

Key words. Space Weather - modeling - validation - software - services

\section{Introduction}

"Space Weather" as a scientific term became widely used near the end of the 20th century. A definition of the discipline that was developed and adopted by the European COST Action 724 states that "Space Weather is the physical and phenomenological state of the natural space environments. The associated discipline aims, through observation, monitoring, analysis and modeling, at understanding and predicting the state of the sun, the interplanetary and planetary environments, and the solar and non-solar driven perturbations that affect them; and also at forecasting and nowcasting the possible impacts on biological and technological systems".

Research efforts in various countries, including the US multi-agency National Space Weather Program and several European initiatives sponsored by the European Space Agency (ESA), the European Commission (EC), and the International Space Environment Service (ISES), have demonstrated that:

- Adverse Space Weather poses a non-negligible threat to humans and modern technological systems and assets on the ground, in the air, and in space;

- Methods to model some aspects of space weather have been developed, although their performance needs to be improved;

- Prediction of the behavior of various Space-Weatherrelated physical parameters is possible and in some cases it has indeed been achieved. However, for many of these physical parameters the prediction accuracy is

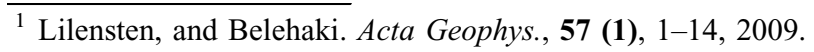

insufficient to allow the transition into reliable operational services and so targeted research and development is needed to address this issue.

To meet further advances, COST Action ES0803 formed an interdisciplinary network between model developers in Europe, warning system developers, operators, users, and policy makers. Through this network the Action aimed at supporting further advances for the development of space weather prediction systems that meet the requirements of the users and at raising awareness and educating the broader public about the implications that adverse space weather phenomena can cause in our daily life. ${ }^{2}$

These objectives have been pursued in the framework of Action ES0803 from 2008 to 2012, with the active participation of more than 80 experts from 24 countries. This paper reports the main scientific results obtained by this Action in three action lines: (1) advances in Space Weather models; (2) recommendations for new Space Weather products and services; (3) dissemination, education, and outreach activities. In the final section, we summarize the main achievements that may have a future impact in the European Space Weather community and we present some plans for the future.

\section{Space Weather prediction models in Europe}

The main objective of this task was to compile and analyze recent advances in the field of Space Weather modeling, to

\footnotetext{
${ }^{2}$ Belehaki, et al. Space Weather - The International Journal of Research and Applications, 7, S03001, 2009.
} 
make recommendations for the assessment and validation of prediction models, and to simulate further upgrades of the models in order to be implemented in an operational environment.

\subsection{Advances in Space Weather modeling}

Recent advances on solar dynamics (the origin of space weather), space plasma processes (the transmission process), and long-term variability of the Sun, interplanetary space, and geospace (the media which modulate it) have been compiled and analyzed for a successful description, nowcasting, and forecasting of the state of the space environment and of specific Space Weather events.

The main findings are reported in a review ${ }^{3}$ on the state of the art for our understanding of phenomena occurring in the solar atmosphere that are due to solar magnetism and are the basis of Space Weather. The review includes combined multi-disciplinary, multi-instrument, multi-wavelength studies of these phenomena, ranging from the very first manifestation of solar active region formation and evolution, to the analysis of explosive phenomena (i.e., flares, erupting prominences, Coronal Mass Ejections), to the study of the interaction of magnetized plasma clouds expelled from the Sun with the interplanetary magnetic field and medium.

The geomagnetic response to solar and interplanetary disturbances is the topic of another review paper ${ }^{4}$ in this topical issue. Following an analysis of their long-term evolution, the Authors discuss short-term responses, where they distinguish between responses of the terrestrial environment to solar activity (and specifically to solar energetic events) and to the solar wind. Geomagnetic responses at low and high latitudes are considered separately. At low latitudes, the evolution of the ring current in both the main and recovery phases is analyzed. At high latitudes, achievements in modeling the coupling between magnetospheric and ionospheric processes are studied, with special attention to the polar caps and field-aligned currents.

The impact of solar activity on the Earth's upper atmosphere has been systematically studied during this Action and the results are summarized in a review paper. $^{5}$ This review is focused on methods based on data-driven analysis. Medium- and long-term ionospheric response to the changes in solar and geomagnetic activity provides results on 27-day response of low latitude ionosphere to solar EUV radiation, response to the recurrent geomagnetic storms, long-term trends in the upper atmosphere, latitudinal dependence of total electron content (TEC) to EUV changes, and statistical analysis of ionospheric behavior during prolonged period of solar activity. Storm-time ionospheric response to the solar and geomagnetic forcing provides results from studies of ionospheric variations induced by recurrent CIR-driven storm, a case-study of polar cap absorption due to an intense CME, and a statistical study of geographic distribution of the so-called E-layer-dominated ionosphere. Empirical models for

\footnotetext{
3 Zuccarello, et al. J. Space Weather Space Clim., 3, A18, 2013, DOI: $10.1051 /$ swsc/2013039.

4 Saiz, et al. J. Space Weather Space Clim., 3, A26, 2013, DOI: $10.1051 /$ swsc/2013048.

${ }^{5}$ Kutiev, et al., J. Space Weather Space Clim., 3, A06, 2013, DOI: $10.1051 / \mathrm{swsc} / 2013028$.
}

forecasting bottomside ionospheric parameters, the electron density and the total electron content, are reviewed. A new method for the retrieval of the basic thermospheric parameters from ionospheric sounding data is also presented. The detection, monitoring, and forecast of ionospheric perturbations in Europe to support GNSS systems has been also reviewed and further studied. ${ }^{6}$

Phenomena in the polar cap ionosphere and the effects on Space Weather were also investigated in this COST Action. ${ }^{7}$ The main part of the work has been directed toward the study of plasma instabilities and scintillations in association with cusp flow channels and polar cap patches, which is considered as a critical knowledge in order to develop forecast models for scintillations in the polar caps. The problem has been approached by multi-instrument techniques that include the EISCAT Svalbard Radar, SuperDARN radars, in-situ rocket measurements, and GPS scintillation measurements.

An important scientific discovery that came out from this Action was the confirmation of detection of the thermospheric auroral red line polarization. ${ }^{8}$ The study was based on the results from a special campaign carried out during the winter in 2010/2011 where the polarization of the red line was measured for the first time at the Polish Hornsund polar base without contamination. Two methods of data analysis are presented to compute the degree of linear polarization (DoLP) and angle of linear polarization (AoLP): one is based on averaging and the other one on filtering. Results are compared and are in qualitative agreement.

Finally, the impact of cosmic rays and solar energetic particles on the Earth's environment has been studied, ${ }^{9}$ with emphasis on numerical models that compute the cosmic ray ionization profiles at a given location and time. Intercomparison of the models, as well as comparison with direct rocket measurements of the atmospheric ionization, validate their applicability for the entire atmosphere and for the different levels of solar activity.

\subsection{Assessment and validation of existing Space Weather research and operational models}

The assessment requires the development of criteria and metrics which can objectively be applied to quantify the performance of the models. Strengths and weaknesses include (but are not limited to) the quantitative accuracy of the model results, the weight of constraints on input parameters and boundary conditions, the validity range of the model, the spatial and temporal scales over which useful predictions are possible when the models are predictive.

The first systematic effort to review assessment and validation efforts of Space Weather models made by European teams was held in the third workshop of the Action (Alcala, Spain,

\footnotetext{
${ }^{6}$ Jakowski, et al. J. Space Weather Space Clim., 2, A22, 2012, DOI: $10.1051 /$ swsc/2012022.

${ }^{7}$ Moen, et al. J. Space Weather Space Clim., 3, A02, 2013, DOI: $10.1051 / \mathrm{swsc} / 2013025$.

${ }^{8}$ Lilensten, et al. J. Space Weather Space Clim., 3, A01, 2013, DOI: $10.1051 / \mathrm{swsc} / 2012023$.

${ }^{9}$ Velinov, et al. J. Space Weather Space Clim., 3, A14, 2013, DOI: $10.1051 / \mathrm{swsc} / 2013036$.
} 
16-17 March 2011). A comprehensive report was compiled ${ }^{10}$ to summarize key issues from the three scientific sessions on: (1) interdisciplinary activities and validation approaches; (2) Space Weather prediction and validation concepts; (3) Space Weather research - models and model support activities. The final conclusion of this activity was presented in the final meeting of the Action ${ }^{11}$ to provide a recommended methodology that can be applied to any Space Weather model. Verification approaches described in their report are taken both from the meteorological and Space Weather community practices. A survey of current Space Weather model verification has been carried out, with focus on models and algorithms developed by institutes participating in COST Action ES0803. The most common approach is to use one, or a few, metrics to verify the models. It is recommended that the more advanced approaches, like the distributions oriented, used in meteorology are also used for the Space Weather model verification.

\subsection{Stimulation of models' upgrades and of the development of reliable computer codes for predicting important Space Weather parameters}

The assessment and validation of selected models led to stimulating the improvement of existing models to enable their implementation in an operational environment.

The outcome of this activity was summarized ${ }^{12}$ to include both the introduction of new models and the improvements to existing codes and algorithms that address the broad range of Space Weather's prediction requirements from the Sun to the Earth. For each case, the following aspects have been considered: input data, output parameters, products or services, operational status of the model, and whether it is supported by validation results, in order to provide a solid basis for future developments. The analysis concerns:

- Advances in Solar Weather predictions: solar activity prediction tools, near-Real-Time detection and tracking of Active Regions and Coronal Holes, Forecasting SEP Events;

- Advances in Geomagnetic predictions: warnings for geomagnetic disturbances, nowcasting and forecasting the $K$ index;

- Advances in Satellite environment predictions: specification of the electron density structure up to geosynchronous heights, thermospheric monitoring, short-time forecast of relativistic electrons at geosynchronous orbit;

- Advances in Communication predictions: improvements in ionospheric mapping techniques, solar wind driven ionospheric forecasting algorithms, geomagnetically driven ionospheric forecasting algorithms;

- Advances in GNSS predictions: ionospheric monitoring based on GNSS data, TEC modeling algorithms;

\footnotetext{
${ }^{10}$ Watermann. Scientific report from the 3rd COST ES0803 workshop "Assessment and validation of space weather models", Alcala Spain, 16-17 March 2011, http://www.costes0803.noa.gr/ documents/meetings/costes080alcala/Scientific\%20report\%20Alcala\% 20workshop.pdf.

${ }^{11}$ Wintoft, et al. COST ES0803 Scientific Products, November 2011, http://www.costes0803.noa.gr/documents/scientificproducts/ SG1.2\%20-\%20report\%20-\%20Nov\%202011.pdf.

${ }^{12}$ Tsagouri, et al. J. Space Weather Space Clim., 3, A17, 2013, DOI: $10.1051 /$ swsc/2013037.
}

- Advances in the prediction of Space Weather effects in the Earth's atmosphere: now- and short-term forecasting of the Chemical Composition of the Middle Atmosphere, Cosmic Ray Ionization Model for Ionosphere and Atmosphere.

\section{Recommendations for new Space Weather products and services}

Recommendations are based on the experience that ES0803 experts already gained from the operation of space weather prediction systems (in Regional Warning Centers or at national research institutes and universities), on systematic contacts with users in the framework of workshops or focused meetings, and on specific state-of-the-art studies. ${ }^{13}$ More specifically, the group that implemented the specific set of activities, had the main objectives of collecting updated users' requirements for Space Weather products and services, and of proposing models that can meet these requirements, based on the work performed for the identification of the new models and the stimulation of their validation. The first systematic effort to perform an organized exchange of ideas with users was held in the second workshop of the Action, in Paris, 22-23 March 2010. Valuable insights into the needs of users in several Space Weather domains including spacecraft operations, aviation, and trans-ionospheric radio propagation have been collected and compiled in a comprehensive report. ${ }^{14}$ Qualitative indication of future needs for Space Weather specification and forecasting information have been provided by spacecraft operators, the EGNOS community, the Galileo system operators, HF users, and aviation regulatory authorities. Details can be found in the web site of the Action (http://www. costes0803.noa.gr/meetings).

Given the specific requirements detailed above and the activities performed by European research groups to improve Space Weather prediction models, the following new products and services for Space Weather have been identified as those that have the potential to meet users' needs:

- Automatic tools to predict solar activity; ${ }^{15}$

- Forecasting SEP Events; ${ }^{16}$

- Warnings for geomagnetic disturbances; ${ }^{17}$

- Nowcasting and forecasting the $K$ index; ${ }^{18}$

- Forecast of relativistic electrons at geosynchronous orbit scintillations of Pc5 type; ${ }^{19}$

\footnotetext{
${ }^{13}$ Heynderickx. COST ES0803 Scientific Products, 2012, http:// www.costes0803.noa.gr/documents/scientificproducts/COSTCatalogue. pdf.

${ }^{14}$ Hapgood, M. Web Proceedings of the 2nd COST ES0803 Workshop, Paris, 22-24 March 2010, http://www.costes0803. noa.gr/documents/meetings/second-workshop-paris-2010/User_session_ summary.pdf.

15 Ahmed, et al. Sol. Phys., 283, 157-175, 2013,

DOI: 10.1007/s11207-011-9896-1.

Colak, and Qahwaji. Sol. Phys., 283, 143-156, 2013,

DOI: $10.1007 / \mathrm{s} 11207-011-9880-9$.

${ }^{16}$ Núñez. Space Weather, 9, S07003, 2011,

DOI: $10.1029 / 2010$ SW000640.

17 Saiz, et al. Ann. Geophys., 26, 3989-3998, 2008.

18 Kutiev, et al. J. Atmos. Sol. Terr. Phys., 71, 589-596, 2009, DOI: 10.1016/j.jastp.2009.01.005.

${ }^{19}$ Degtyarev, et al. Geomag. Aeron., 50 (7), 885-893, 2010.

Degtyarev, et al. Adv. Space Res., 43 (5), 829-836, 2009, DOI: $10.1016 /$ j.asr.2008.07.004.

Potapov, and Polyushkina. Geomag. Aeron., 50 (8), 28-34, 2010.
} 
- 3D specification of the electron density structure up to GNSS heights; ${ }^{20}$

- System for thermospheric monitoring; ${ }^{21}$

- Nowcasting and forecasting models of ionospheric parameters at the peak height with an accuracy range, ${ }^{22}$

- Maps of vertical TEC calculated in real time with activity index ${ }^{23}$

- Real-time GIC analyzer software $;^{24}$

- Advanced data analysis techniques to correlate satellite anomaly data for different orbits with various characteristics of space weather, using rich databases of anomalies recordings; ${ }^{25}$

\section{The education and outreach programme of COST ES0803}

The educational and outreach activities held in the framework of this Action are described in detail in a comprehensive report. $^{26}$ In the following, we provide a brief presentation of some key activities.

\subsection{Educational activities}

The Action organized two international schools: (1) the International Advanced School on Space Weather Modelling and Applications that aimed at providing the scientific knowledge for monitoring, modeling, and predicting Space Weather but with special attention to the applied aspects of Space Weather, i.e., to the monitoring and modeling resources based on advanced data handling for Space Weather. The school was organized at ICTP (Trieste, Italy) in collaboration with INAF, and the EC FP7 Project SOTERIA; (2) the First European School on fundamental processes in Space Weather: a challenge in numerical modeling that trained the students on basic processes for Space Weather, on the theory of magnetic reconnection and instabilities in space, and on simulations and computing. The school was organized in Spineto (Italy) together with the EC FP7 SWIFF consortium.

Our experience from the organization of the two training schools shows that training schools are powerful means for dissemination and education, very effective in raising awareness on the subject, attract new scientists to the field, and are also very effective in synergizing new collaborations.

\footnotetext{
${ }^{20}$ Belehaki, et al. J. Space Weather Space Clim., 2, A20, 2012, DOI: $10.1051 / \mathrm{swsc} / 2012020$.

Kutiev, et al. J. Space Weather Space Clim., 2, A21, 2012, DOI: $10.1051 /$ swsc/2012021.

${ }^{21}$ Mikhailov, et al. J. Space Weather Space Clim., 2, A03, 2012, DOI: $10.1051 /$ swsc/2012002.

22 Tsagouri, I. J. Space Weather Space Clim., 1, A02, 2011, DOI: $10.1051 / \mathrm{swsc} / 2011110003$.

Pietrella, M. Ann. Geophys., 30, 343-355, 2012,

DOI: 10.5194/angeo-30-343-2012.

Hoque, and Jakowski. Ann. Geophys., 30, 787-809, 2012, DOI: 10.5194/angeo-30-797-2012.

${ }^{23}$ Bergeot, et al. GPS Solutions, 15 (2), 171, 2011,

DOI: 10.1007/s10291-010-0181-9.

${ }^{24}$ Viljanen. Space Weather, 9, S07007, 2011, DOI: $10.1029 / 2011$ SW000680.

${ }^{25}$ Dorman, L. COST ES0803 Final Report on-line version, 2012, http://www.costes0803.noa.gr/documents/scientificproducts/Space\% 20Weather\%20effects\%20on\%20satellites\%20-Dorman.pdf.

${ }^{26}$ Vanlommel, et al. J. Space Weather Space Clim., 4, A05, 2014, DOI: $10.1051 / \mathrm{swsc} / 2014002$.
}

\subsection{Outreach activities}

The outreach programme of the Action was based on a comprehensive set of outreach materials that can be used by experts willing to organize outreach activities in their own country. Main contributions to this material come from the programme I Love my Sun, and the Planeterrella experiment.

The I Love My Sun programme is a COST example of international collaborative outreach. "I Love My Sun" is an educational outreach tool that has been developed for schoolchildren in the approximate age range of 7 through 11 years. The main objective of this tool is to make children aware of Space Weather, the Sun, Sun-Earth relations and how they, the children, are part of this global picture. Children are given a lecture about the Sun. The lecture is preceded and followed by the children drawing a picture of the Sun. Several events have been organized from COST ES0803 experts in Turkey, Belgium, Ukraine and Serbia, and the results are presented and analyzed.

The Planeterrella is another important tool for public outreach. ${ }^{28}$ It is based on the Terrella experiment created by the Norwegian scientist K. Birkeland. By this experiment, Birkeland demonstrated the making of auroras. The Terrella has been greatly improved and constitutes now a new experiment called the Planeterrella. It allows to picture many phenomena occurring in the space environment. It is flexible and attractive. This experiment had initially been developed to be small and modest, shown to local people by a demonstrator. It met a great success which relies on two strong points: (i) there is no patent on it and the plans are given freely to any public institution and (ii) the advertisement does not rely on press release, books, or web sites but mainly on national and European scientific networks such as COST ES0803.

Today, nine Planeterrellas are operating or under construction in four different countries, and more are foreseen. In five years, about 50,000 people (and much more on TVs) in Europe could directly see the making of auroras, picture the space environment, and get an introduction to Space Weather with this experiment.

\section{Summary and conclusions}

The main scientific achievements of this COST Action are respectively: (1) the recommended methodology for the validation of Space Weather models; (2) the stimulation of improvements of models based on assessment results; (3) the collection of updated information concerning users' requirements and recommendations for new products and services that can be derived from advanced Space Weather algorithms.

In parallel, networking and coordination activities organized by the Action further supported the establishment of the European Space Weather scientific community with two important achievements:

- The organization for four consecutive years of the European Space Weather Weeks (ESWW), in collaboration with ESA and STCE (ESWW6 to ESWW9). ESWWs are now an international event attracting a large audience of more than

\footnotetext{
${ }^{27}$ Tulunay, et al. J. Space Weather Space Clim., 3, A04, 2013, DOI: $10.1051 / \mathrm{swsc} / 2013026$.

${ }^{28}$ Lilensten, et al. J. Space Weather Space Clim., 3, A07, 2013, DOI: $10.1051 / \mathrm{swsc} / 2013029$.
} 


\section{A. Belehaki et al.: COST Action ES0803 Special Issue Preface}

300 participants consisting of scientists, users, policy makers, all concerned about Space Weather;

- The establishment of the international scientific peer review open access Journal of Space Weather and Space Climate (SWSC) published by EDP Sciences. $^{29}$ The journal accepted the first submissions (http:// www.swsc-journal.org/) in 2011. Today SWSC is a well-established journal with more than 90 scientific papers published to date. The first Impact Factor released by Thomson Reuters in 2014 is 2.519 .

Future developments in the field will have to take into account the general requirements of Space Weather users that concern:

(a) Continuous improvements of Space Weather prediction models to tailor the accuracy and delivery method according to the continuously evolving needs of users;

(b) Standardization of the Space Weather products and services to encourage the use by a wide user community;

(c) Assurance of long-term access to data;

(d) Use of validated models to provide quality indicators to the user;

(e) Development of tools to improve operators' ability to explore data through the use of advanced data analysis techniques; (f) Increasing awareness of Space Weather and users' education about possible effects and what can be done for warning and mitigation.

Valuable tools that support these requirements are the data e-infrastructures that have been developed or are under development by European space science communities, able to provide access to homogenized and standardized space data through e-science tools. These data platforms should be systematically exploited by the European Space Weather community to further meet the requirements of the users and to provide services assured on the long term.

Acknowledgements. We acknowledge the excellent collaboration from the Leaders of the Working Groups Dr. Jürgen Watermann, Prof. Mike Hapgood, and Dr. Petra Vanlommel. We are also extremely grateful to Prof. Ronald Van der Linden, who supported this Action as Grant Holder, as Co-Organizer of the ESWW through STCE, and as Co-Leader of Working Group 2. Special thanks are due to Dr. Jean Lilensten for his enthusiastic contribution and substantial support for the establishment of the Journal of Space Weather and Space Climate. Finally, we would like to acknowledge the support from Dr. Carine Petit through her role as Scientific Officer of the Action from 2008 to 2011 and the support from Prof. Sylvain Joffre for stimulating discussions while he was Chair of the DC of the ESSEM.

Cite this article as: Belehaki A., M. Messerotti \& M. Candidi. Developing Space Weather products and services in Europe - Preface to the Special Issue on COST Action ES0803. J. Space Weather Space Clim., 4, E1, 2014, DOI: 10.1051/swsc/2014032.

${ }^{29}$ Belehaki, and Lilensten. J. Space Weather Space Clim., 3, A13, 2013, DOI: $10.1051 / \mathrm{swsc} / 2013035$.

Lilensten, and Belehaki. J. Space Weather Space Clim., 1 (1), E01, 2011, DOI: $10.1051 / \mathrm{swsc} / 2011002$. 